\title{
Fitorremediación de mercurio a partir de elodea sp.
}

\author{
María Consuelo Jaramillo $\mathrm{F}$. \\ Universidad de San Buenaventura \\ mariac.jaramillo@usbmed.edu.co
}

\author{
Luisa Fernanda Zapata 0. \\ Universidad de San Buenaventura \\ luisa.zapata@tau.usbmed.edu.co
}

\author{
Tatiana Marulanda L. \\ Universidad de San Buenaventura \\ tmarulandalopez@gmail.com
}

(Tipo de Artículo: Investigación. Recibido el 03/07/2015. Aprobado el 10/09/2015)

Resumen. El mercurio es un contaminante derivado de la minería, el cual es altamente tóxico para plantas, animales y el ser humano. El mercurio se bioacumula en plantas y peces que son de consumo humano. En esta investigación se evaluó la capacidad de biorremediación del ion mercurio por la planta macrofita Elodea sp. Tallos de $35-36 \mathrm{~cm}$ de la planta fueron sometidos a diferentes concentraciones de solución de $\mathrm{HgCl}_{2}$. Las concentraciones evaluadas fueron de $0,39 \mathrm{mg} / \mathrm{ml}, 1,55 \mathrm{mg} / \mathrm{ml}$ y de $6,25 \mathrm{mg} / \mathrm{ml}$, aunque la planta Elodea sp mostró un gran desempeño en las tres soluciones, con la concentración de 1,55mg/ml obtuvo un porcentaje de remoción del 100\%, lo cual lo convierte en las condiciones a las que debe ser removido el mercurio.

Palabras clave. Absorción, adsorción, cuerpos de agua, Elodea sp, Fitorremediación, macrofita.

\section{Phytoremediation by elodea sp.}

\begin{abstract}
Mercury is a contaminant from mining, which is highly toxic to plants, animals and humans. Mercury is bioaccumulated in plants and fish of human consumption. This research evaluated bioremediation capacity of mercury ion by the aquatic macrophyte Elodea sp. Stems of 35-36 cm of plants has been evaluated at different concentrations of $\mathrm{HgCl}_{2}$ solution. The evaluated concentrations were $0,39 \mathrm{mg} / \mathrm{ml}, 1,55 \mathrm{mg} / \mathrm{ml}$ and of $6,25 \mathrm{mg} / \mathrm{ml}$, although the Elodea sp plant showed high performance in the three solutions, but the plant showed $100 \%$ of removing of mercury at $1,55 \mathrm{mg} / \mathrm{ml}$ concentration, therefore this concentration was become in suitable conditions for removing mercury.
\end{abstract}

Keywords. Absorption, adsorption, water bodies, Elodea sp,, Phytoremediation, macrophyte.

\section{INTRODUCCIÓN}

El mercurio es un metal pesado que a temperatura ambiente se encuentra en forma líquida, es soluble solo en soluciones oxidantes y además contiene un alto nivel de toxicidad el cual es perjudicial en plantas, animales y la salud humana [1]. El mercurio se encuentra en forma natural, pero en las últimas décadas se ha aumentado su concentración en el ambiente debido a factores antrópicos causados por la actividad industrial y minera, acumulándose en el aire, suelo y agua. Las industrias aprovechan este metal en la manofactura de equipos electrónicos y científicos como baterías, lámparas, termómetros y barómetros, además es utilizado para la elaboración de pesticidas, conservadores de semillas, pinturas, y algunos cosméticos [2] [3]. El medio más común en el que se encuentra este metal es en el agua, y por lo tanto, es la forma en la que puede ser transportado con mayor facilidad a cualquier organismo vivo, e intervenir en el proceso de bioacumulación; en cuanto al mercurio que se encuentra en el suelo, no es tan significativa su concentración, ya que a través de ciertas plantas puede ser removido con facilidad, algunas de las plantas más utilizadas para este fin es el guaramo y Jatropha curcas ubicadas por lo general en bosques tropicales [4] [5]; además, mucha parte del mercurio presente en el suelo puede formar complejos con la arcilla, limitando en gran medida la movilidad que este tenga por el suelo [6]. Es por eso que la mayor preocupación de los últimos tiempos respecto a la contaminación por mercurio, recae en las concentraciones contenidas en algunos cuerpos de agua, y a partir del cual pueden tener contacto directo algunos organismos vivos transfiriéndose por medio de la alimentación de un ser vivo a otro(s), siendo entonces este el tema y motivo de investigación a través del cual se pretende estudiar qué capacidad tienen las plantas microfitas para remover mercurio en cuerpos acuosos.

Las Plantas macrofitas son aquellas plantas adaptadas a la vida en el medio acuático y que gracias a esta capacidad pueden presentar varios beneficios como [7]: puede utilizarse para alimentación humana, del ganado, de peces y otros animales acuáticos; pueden ser utilizadas como fertilizantes; pueden usarse para purificación del agua; para uso medicinal y en cosmetología; para producción de celulosa; como fuente de producción de bio-gas.

En este trabajo se estudió la macrofita Elodea sp en el tratamiento de aguas contaminadas con mercurio. La planta macrofita Elodea $s p$ es una planta acuática que puede reproducirse con facilidad en algunas partes del mundo, tanto así que puede considerarse una plaga al obstruir los sistemas de drenaje. Crece con largos tallos, con hojas ovaladas y las raíces pueden emerger en cualquier parte del tallo adhiriéndose con facilidad y rápidamente a un soporte (suelo) [8].

De igual manera existen varios estudios relacionados con la remoción de mercurio en diferentes cuerpos de 
agua, utilizando el proceso de fitorremediación sostenible para hacer frente al reto de la bioacumulaciòn de metales pesados en sitios contaminados [9], pero utilizando diferentes especies de plantas macrofitas como Pistia stratiotes y Azolla pinnata, son plantas acuáticas utilizadas para reducir el mercurio presente en un afluente cercano a una mina. El porcentaje de eliminación de $P$. stratiotes $y$ A. pinnata fue del $80 \%$ y $68 \%$, respectivamente. Indicando muy buenos resultados en estas plantas [10]-[12].

\section{METODOLOGÍA}

La metodología se siguió de acuerdo a las referencias [13]-[19].

\subsection{Material vegetal: Elodea sp.}

La planta fue adquirida en un acuario de la ciudad de Medellín, y se dispuso en el laboratorio en un recipiente destapado con agua y arena hasta la utilización en el ensayo. Para el ensayo se tomaron tallos entre $35-36 \mathrm{~cm}$ de largo y se introdujeron en los elermeyers con la solución a tratar

\subsection{Curva de calibración de la solución de $\mathrm{HgCl} 2$}

Se preparó una solución stock de $12.5 \mathrm{mg} / \mathrm{ml}$ en agua. Se realizaron 5 diluciones sucesivas $(6,25 \mathrm{mg} / \mathrm{ml} ; 3,125$ $\mathrm{mg} / \mathrm{ml} ; 1,55 \mathrm{mg} / \mathrm{ml} ; 0,78 \mathrm{mg} / \mathrm{ml} ; 0,39 \mathrm{mg} / \mathrm{ml}$ ) para la curva de calibración.

\subsection{Método analítico}

La curva de calibración se realizó en el espectrofotómetro (GENESYS 10S UV-Vis, Thermo SCIENTIFIC) tomando lecturas da la absorbancia cada 30 minutos por 2 hr a $28^{\mathrm{a}} \mathrm{C}$. la concentración de mercurio fue medida a través de la absorbancia a $253 \mathrm{~nm}$.

\subsubsection{Ensayo de fitorremediación.}

En elermeyers de $250 \mathrm{ml}$ se prepararon $100 \mathrm{ml}$ de las concentraciones $6,25 \mathrm{mg} / \mathrm{ml} ; 1,55 \mathrm{mg} / \mathrm{ml} ; 0,39 \mathrm{mg} / \mathrm{ml}$ y control a las cuales se les trató con la planta. Cada concentración y control se preparó por triplicado.

\subsubsection{Determinación de metal absorbido por la planta.}

Se tomaron las plantas de cada reactor, se lavaron con agua destilada, con una solución de $0.1 \%$ de EDTA, y de nuevo con agua. Las plantas se secaron a $60^{\circ} \mathrm{C}$ por 4 horas, para obtener $58 \mathrm{gr}$ de planta seca. La planta se puso a digestión por 30 minutos con $10 \mathrm{ml}$ de agua y $7 \mathrm{ml}$ de $\mathrm{HNO}_{3}$ concentrado. Se filtró cada una de las plantas y se realizó la lectura de absorbancia tomando como blanco el control.

\subsubsection{Análisis estadístico}

Los resultados son presentados como la media \pm SEM del triplicado, los datos fueron analizados usando el programa Graph-Pad 5.0 software 2007, San Diego, CA,
USA. Un valor de P menor de 0,05 fue considerado significativo.

\section{RESULTADOS Y DISCUSIÓN}

\subsection{Curva de calibración}

La Fig. 1 muestra la curva de calibración de las soluciones de $\mathrm{HgCl}_{2}$ a $253 \mathrm{~nm}$. De esta se obtuvo la ecuación (1)

$$
Y=0,10222 X+0,05396
$$

Donde $Y$ representa la absorbancia de la muestra y $X$ la concentración en unidades de $\mathrm{mg} / \mathrm{ml}$

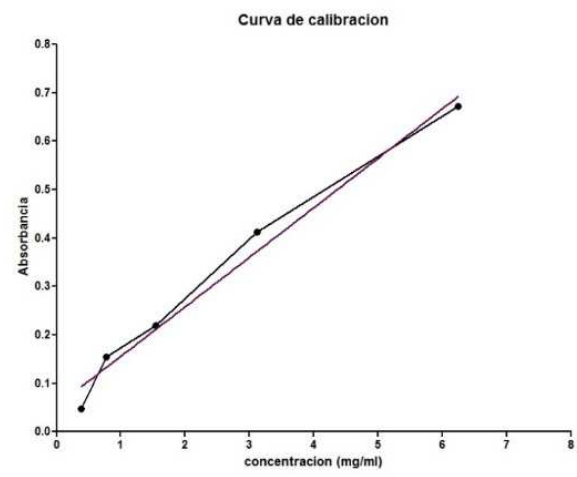

Fig. 1. Curva para la calibración

La línea mostró un coeficiente de correlación de 0,98 y un $\mathrm{P}=0,0011$, como es menor a 0,05 fue considerado como significativo.

\subsection{Curvas de remediación de mercurio para las diferentes concentraciones de $\mathrm{HgCl} 2$}

La Fig. 2 muestra el comportamiento descendente que tiene la curva de concentración $0,39 \mathrm{mg} / \mathrm{ml}$, respecto al tiempo en el que fueron tomadas las muestras, es decir, a medida que transcurrió el tiempo la concentración de mercurio se redujo.

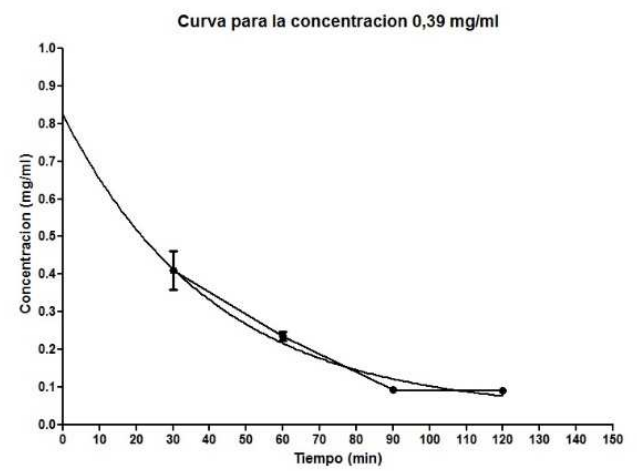

Fig. 2. Curva de fitorremediación de Mercurio a la concentración de $0,39 \mathrm{mg} / \mathrm{ml}$

Después de los 90 minutos la concentración de mercurio en agua fue constante $0,093 \mathrm{mg} / \mathrm{ml}$, mostrando la saturación de la planta con el metal 
El comportamiento que tuvo la Elodea $s p$ en la concentración $1,55 \mathrm{mg} / \mathrm{ml}$ se muestra en la Fig. 3, donde se observa el comportamiento descendiente a través del tiempo.

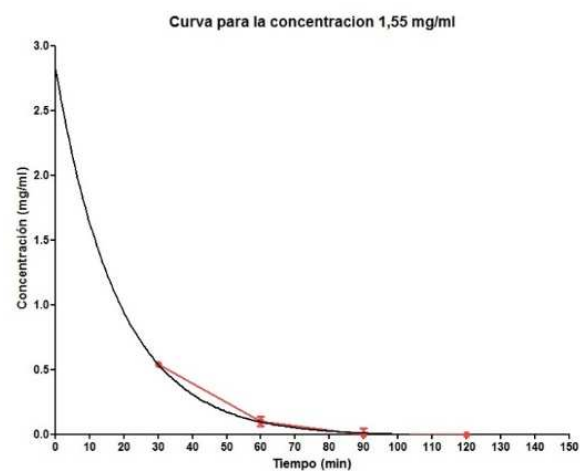

Fig. 3. Curva de fitorremediación de Mercurio a la concentración de $1,55 \mathrm{mg} / \mathrm{ml}$.

Se observa que después de los 60 minutos la concentración se redujo a $100 \mathrm{mg} / \mathrm{ml}$, y a los 90 minutos la planta ya había alcanzado la remediación total del mercurio en la solución.

La Fig. 4 muestra la curva de remediación en la concentración $6,25 \mathrm{mg} / \mathrm{ml}$. A pesar de que esta fue la concentración más alta utilizada dentro de este estudio, pudo observarse la tendencia descendente en el tiempo.

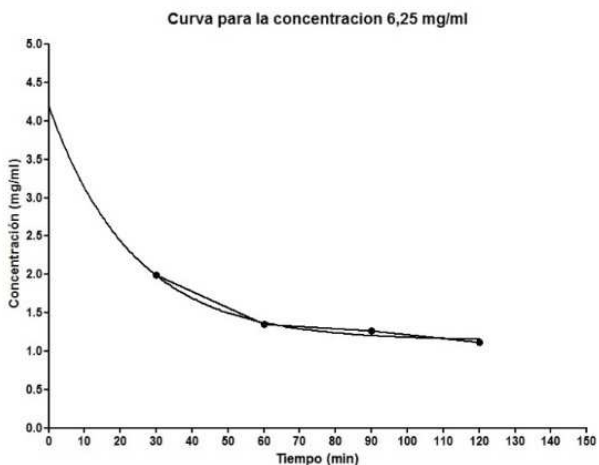

Fig. 4. Curva de fitorremediación de Mercurio a la concentración de $6,25 \mathrm{mg} / \mathrm{ml}$.

La curva muestra un comportamiento constante después de los 60 minutos donde la concentración se redujo a $1,35 \mathrm{mg} / \mathrm{ml}$ y a los 120 minutos la concentración era $1,18 \mathrm{mg} / \mathrm{ml}$, manteniéndose constante.

\subsection{Porcentajes de remoción de mercurio respecto a cada concentración.}

En la tabla 1 se observan los porcentajes de remoción de mercurio en cada una de las soluciones. La Elodea $s p$ tuvo un mejor desempeño de remoción en la solución de concentración $1,55 \mathrm{mg} / \mathrm{ml}$, con un porcentaje de remoción del $100 \%$, a esta concentración la planta se encuentra en equilibrio osmótico para lograr una máxima remediación, y optimizar y asegurar la descontaminación de un medio acuático.

TABLA 1

Porcentaje de remoción de mercurio de acuerdo a su concentración.

\begin{tabular}{cc}
\hline $\begin{array}{c}\text { Concentración } \\
(\mathrm{mg} / \mathrm{ml})\end{array}$ & $\begin{array}{c}\text { Porcentaje de mercurio } \\
\text { removido de la solución (\%) }\end{array}$ \\
\hline 0.39 & 76.5 \\
1.55 & 100.0 \\
6.25 & 82.1 \\
\hline
\end{tabular}

En la concentración de $0.39 \mathrm{mg} / \mathrm{ml}$ se observa un porcentaje de remediación del $76.5 \%$, y a $6.25 \mathrm{mg} / \mathrm{ml} \mathrm{la}$ remediación fue del $82.1 \%$. Este comportamiento podría sugerir que existe un equilibrio donde la planta remedia sin que haya toxicidad celular. A concentraciones mayores de $1,55 \mathrm{mg} / \mathrm{ml}$ fue toxico para la planta y a menor concentración, no se optimiza la capacidad de remediar de la planta.

\subsection{Porcentaje de mercurio absorbido por la planta.}

Es importante conocer la cantidad de mercurio que absorbió la planta dentro de su estructura respecto a la cantidad que fue removida de la solución, debido a que de esa manera se conoce cuál fue el proceso de remoción de mercurio que tuvo la planta, es decir, de acuerdo al porcentaje que fue removido de la solución puede determinarse la cantidad de mercurio que fue adsorbido y/o absorbido por la planta. En la Tabla 2 se muestran cada uno de los porcentajes absorbidos y adsorbidos por la planta, notándose que bajo una concentración de 1,55 $\mathrm{mg} / \mathrm{ml}$ la Elodea $s p$ tuvo mayor capacidad de absorción que de adsorción.

TABLA 2

Porcentaje de mercurio retenido en la planta

\begin{tabular}{ccc}
\hline $\begin{array}{c}\text { Concentración } \\
(\mathrm{mg} / \mathrm{ml})\end{array}$ & $\begin{array}{c}\text { Porcentaje } \\
\text { absorbido por la } \\
\text { planta (\%) }\end{array}$ & $\begin{array}{c}\text { Porcentaje } \\
\text { adsorbido por la } \\
\text { planta (\%) }\end{array}$ \\
\hline 0.39 & 8.4 & 68.2 \\
1.55 & 77.4 & 22.6 \\
6.25 & 9.2 & 72.9 \\
\hline
\end{tabular}

A concentraciones de $0.39 \mathrm{mg} / \mathrm{ml}$ el porcentaje de absorción fue $8.4 \%$ y a $6.25 \mathrm{mg} / \mathrm{ml}$ fue absorbido el $9.2 \%$. Esto muestra que a la concentración de $1.55 \mathrm{mg} / \mathrm{ml} \mathrm{la}$ planta absorbe en mayor proporción, es decir, es la concentración donde ocurre el paso del ion mercurio a través de la membrana celular; a concentraciones más bajas o más altas, la membrana celular no es activada.

La planta usa como proceso de remediación la adsorción, pero el porcentaje de remediación aumenta si el metal es absorbido, pero para que ocurra la absorción la planta debe estar a las concentraciones adecuadas.

\section{TRABAJOS FUTUROS}

La contaminación de mercurio en el agua es un tema muy amplio y complejo en cuanto a las consecuencias que tiene para cualquier tipo de organismo. Gracias a esta investigación se pudo comprobar que la planta Elodea $s p$ puede remediar cuerpos de agua de la contaminación 
producida por el mercurio. Esta investigación es la base para la aplicación de plantas invasoras como potenciales medios de remediación de metales pesados de cuerpos acuosos [20]-[22].

\section{CONCLUSIONES}

La Elodea $s p$ tiende a remover mercurio a las concentraciones evaluadas. Según los resultados mostrados, el mecanismo usado por la planta para remediar el mercurio es a través de la absorción y adsorción del metal. Inicialmente la planta adsorbe el metal, y dependiendo de la concentración, la planta absorbe el metal. A concentraciones muy altas se presenta toxicidad, pero el mecanismo de adsorción se muestra en estas condiciones, aunque el porcentaje de remediación es menor, por lo tanto el factor limitante de la remediación es la concentración a la que es tóxica el metal. Esta investigación muestra a la Elodea sp como una planta macrófita potencialmente utilizable como fuente de descontaminación de aguas contaminadas con mercurio.

El impacto, daño y toxicidad del mercurio sigue siendo significativo y peligroso para la vida, lo que justifica el esfuerzo en las investigaciones sobre el tratamiento de estos los cuerpos acuosos [23].

\section{AGRADECIMIENTOS}

Los autores reconocen los agradecimientos a la Universidad de San Buenaventura por el apoyo a la investigación, igualmente a la laboratorista Jessica Henao Hernández por la asistencia durante la elaboración de la investigación.

\section{REFERENCIAS}

[1] A. Ullah; S. Heng; M. F. Hussain Munis; S. Fahad and X. Yang. "Phytoremediation of heavy metals assisted by plant growth promoting bacteria: a review". Environmental and Experimental Botany, Vol. 117, pp. 28-40, 2015.

[2] Ambientum, "El mercurio como elemento contaminante," Ambientum, 2001. [En línea]. Available: http://www.ambientum.com/revista/2001_18/2001_18_ATMOSFE RA/LMRCRCML2.htm. [Último acceso: 0906 2015].

[3] P. Miretzky; A. Saralegui and A. Fernández Cirelli. "Aquatic macrophytes potential for the simultaneous removal of heavy metals (Buenos Aires, Argentina)". Chemosphere, Vol. 57, pp. 997-1005, 2004.

[4] J. V. Vidal Durango. "Remediación de suelos contaminados con mercurio utilizando guarumo,2 Revista Científica Ingeniería y Desarrollo, no 27, p. 1, 2010.

[5] J. M. Negrete; J. D. Hernandez, J. P. Hernandez y S. D. Jesus Olivero Verbel, "Phytoremediation of mercury- Contaminated soils by Jatropha curcas," Chemosphere , vol. 127, pp. 58-63, 2015.

[6] X. G. Martinez, , Universidad Autonoma de Bracelona, "El mercurio como contaminante global," 08 2004. [En línea]. Available: http://www.tdx.cat/bitstream/handle/10803/3174/xgm1de1.pdf;jse ssionid=0B7BA87966545FD4DCC931DE91DB08E5.tdx1?seque nce=1. [Último acceso: 1106 2015].

[7] Arreghini, S. "Plantas acuàticas (macrofitas)," [En línea]. Available http://www.cricyt.edu.ar/enciclopedia/terminos/PlantAcuat.htm. [Último acceso: 0906 2015].
[8] Red Naturaleza, "El portal de la naturaleza," Red de Naturaleza., [En línea]. Available: http://www.rednaturaleza.com/peces/plantas-de-acuario-elodeadensa. [Último acceso: 0906 2015].

[9] V. C. Pandey; D. N. Pandey and N. Singh. "Sustainable phytoremediation based on naturally colonizing and economically valuable plants,» Journal of cleaner Production, Vol. 86, pp. 37-39, 2015.

[10] V. K. Mishra, B. D. Tripathi and K.-H. Kim, «Removal and accumulation of mercury by aquatic macrophytes from an open cast coal mine effluent, J Journal of Hazardous Materials., vol. 172, pp. 749-754, 2009.

[11] H. Ali; E. Khanand M. A. Sajad. «Phytoremediation of heavy metals-Concepts and applications». Chemosphere, Vol. 91, pp. 869-881, 2013

[12] M. A. Kahkonen; M. Pantsar-Kallio and P. K. G. Manninen, «Analysing heavy metal concentrations in the different parts of Elodea Canadensis and surface sediment with pca in two boreal lakes in Southem Finland,» Chemosphere, Vol. 35, pp. 2645-2656, 1997.

[13] M. A. Kankonen and P. K. G. Manninen. «The uptake of nickel and chromium from water by elodea canadensis at different nickel and chromium exposure levels,» Chemosphere, Vol. 36, pp. 13811390, 1998.

[14] A. Fritioff, and M. Greger. «Fate of cadmium in Elodea Canadensis,» Chemosphere, Vol. 67, pp. 365-375, 2007.

[15] R. Olette; M. Couderchet ; S. Biagianti and P. Eullaffroy. "Toxicity and removal of pesticides by selected arquatic plants," Chemosphere, Vol. 70, pp. 1414-1421, 2008.

[16] E. B. McGregor ; K. R. Solomons and M. L. Hanson, «Effects of planting sytem design on the toxicological sensitivity of Myriophyllum spicatum and Elodea canadensis to atrazine,» Chemosphere, Vol. 73, pp. 249-260, 2008.

[17] M. G. Maleva; G. F. Nekrasova; P. Malec; M. N. V. Prasad and K. Strzalka, «Ecophysiological tolerance of Elodea canadensis to nickel exposure,» Chemosphere, Vol. 77, pp. 392-398, 2009.

[18] N. Regier; F. Larras; A. Garcia Bravo; V. G.Ungureanu; D. Amouroux and C. Cosio. «Mercury bioarccumulation in the aquatic plant Elodea nuttallii in the field and in microcosm: Accumulation in shoots from the water might involve copper transpoters,» Chemosphere, Vol. 90, pp. 595-602, 2013.

[19] L. Bondareva; I. Vlasova; O. Moglinaya; A. Bolsunovsky and S. Kalmykov. «Microdistribution of $241 \mathrm{Am}$ in structures of submerged macrophyte elodea canadensis growing in the Yenisei River," Jornal of Environmental Radioactivity, Vol. 101, pp. 16-21, 2010.

[20] M. M. Veiga; G. Angeloci-Santos and J. A. Meech. «Review of barriers to reduce mercury use in artisanal gold mining". The Extractive Industries and Society, Vol. 1, pp. 351-361, 2014.

[21] M. E. Finster; M. R. Raymond; M. A. Scofield and K. P. Smith. "Mercury-impact scrap metal: Source and nature of the mercury». Journal of Environmental Management, Vol. 161, pp. 303-308, 2015.

[22] Surriya, O. S. Sarah, K. Waqar y A. G. Kazi. «Phytoremediation of soils: Prospects and Challenges," Soil Remediation and plants , Vol. 1, pp. 1-36, 2015.

[23] A. H. Fostier; J. J. Melendez-Perez and L. Richter. «Litter mercury deposition in the Amazonian rainforest». Environmental Pollution, Vol. 206, pp. 605-610, 2015 